\title{
POSITIVE DEFINITE OPERATOR SEQUENCES
}

\author{
T. BISGAARD
}

(Communicated by Palle E. T. Jorgensen)

\begin{abstract}
An example is given of a linear mapping from $C[x]$ to $\mathbf{M}_{2}(\mathbf{C})$ which is positive but not completely positive. It is shown that a positive linear mapping from $\mathbf{C}[x]$ to $\mathbf{B}(\mathscr{H})$ is completely positive if certain scalar moment sequences associated with it are determinate.
\end{abstract}

Suppose $\mathscr{A}$ is a complex algebra with involution, $\mathscr{B}$ is a $C^{*}$-algebra, and $L$ is a linear mapping from $\mathscr{A}$ to $\mathscr{B}$. We say $L$ is positive if $L\left(a^{*} a\right) \geq 0$ for each $a \in \mathscr{A}$. For each integer $n$ the set $\mathbf{M}_{n}(\mathscr{A})$ of matrices of order $n$ with entries in $\mathscr{A}$, with matrix multiplication and the involution $\left(a_{j k}\right)^{*}=\left(a_{k j}^{*}\right)$, is again a complex algebra with involution and $\mathbf{M}_{n}(\mathscr{B})$ is a $C^{*}$-algebra. The mapping $L$ is said to be completely positive if for each $n$ the mapping $\left(a_{j k}\right) \rightarrow\left(L\left(a_{j k}\right)\right)$ from $\mathbf{M}_{n}(\mathscr{A})$ to $\mathbf{M}_{n}(\mathscr{B})$ is positive. For studies of such mappings we refer to $[2,6-9,11,12]$. See [5] for a fairly recent survey.

Evidently, every completely positive mapping is positive. The converse, however, is false, even if $\mathscr{A}$ is assumed to be a $C^{*}$-algebra, as evidenced by the example of Arveson [2, p. 169] that the transpose operation on $\mathbf{M}_{2}(\mathbf{C})$ is positive but not completely positive. It is known that if $\mathscr{A}$ is a commutative $C^{*}$-algebra or if $\mathscr{B}$ is commutative, then every positive linear mapping from $\mathscr{A}$ to $\mathscr{B}$ is completely positive (see $[2,11])$. The main aim of the present note is to show that commutativity of $\mathscr{A}$ (without the condition that $\mathscr{A}$ be a $C^{*}$-algebra) does not imply that every positive linear mapping from $\mathscr{A}$ to $\mathscr{B}$ is completely positive. In fact, the conclusion fails even if $\mathscr{A}=\mathbf{C}[x]$, the polynomials in one indeterminate with complex coefficients, and $\mathscr{B}=\mathbf{M}_{2}(\mathbf{C})$. A counterexample is given in Theorem 1.

Theorem 2 provides a sufficient condition, in terms of determinacy of certain scalar moment sequences, for a positive linear mapping defined on $\mathbf{C}[x]$ to be completely positive. An application shows that a positive linear mapping $L: \mathbf{C}[x] \rightarrow \mathbf{B}(\mathscr{H})$ is completely positive if only $\left\|L\left(x^{n}\right)\right\|$ does not grow too rapidly (i.e., not much faster than $n^{n}$ ) as $n \rightarrow \infty$. Finally, Theorem 3 is a strengthening of Theorem 1, stating that a positive linear mapping from $\mathbf{C}[x]$

Received by the editors April 12, 1991 and, in revised form, June 3, 1991 and November 12, 1992.

1991 Mathematics Subject Classification. Primary 44A60. 
to $\mathbf{B}(\mathscr{H})$ may fail to be completely positive even if many of the associated scalar moment sequences are determinate.

A sequence $\left(s_{n}\right)_{n \geq 0}$ of bounded operators on a Hilbert space $\mathscr{H}$ is said to be positive definite if $\sum_{j, k=0}^{n} \bar{c}_{j} c_{k} s_{j+k} \geq 0$ for all $n \geq 0$ and $c_{0}, \ldots, c_{n} \in \mathbf{C}$, and of positive type if $\sum_{j, k=0}^{n}\left\langle s_{j+k} \xi_{k}, \xi_{j}\right\rangle \geq 0$ for all $n \geq 0$ and $\xi_{0}, \ldots, \xi_{n} \in \mathscr{H}$ (cf. [4, 4.1.7] or [13]). Note that $\left(s_{n}\right)$ is positive definite if and only if for each $\xi \in \mathscr{H}$ the scalar sequence $\left(\left\langle s_{n} \xi, \xi\right\rangle\right)$ is positive definite. Sequences $\left(s_{n}\right)$ in $\mathbf{B}(\mathscr{H})$ are in a one-to-one correspondence with linear mappings $L: \mathbf{C}[x] \rightarrow$ $\mathbf{B}(\mathscr{H})$, given by $L\left(x^{n}\right)=s_{n}$. Such a sequence $\left(s_{n}\right)$ is positive definite if and only if the corresponding mapping $L$ is positive and is of positive type if and only if $L$ is completely positive. We identify $\mathbf{B}\left(\mathbf{C}^{n}\right)$ with $\mathbf{M}_{n}(\mathbf{C})$.

Theorem 1. There is a positive linear mapping from $\mathbf{C}[x]$ to $\mathbf{M}_{2}(\mathbf{C})$ which is not completely positive.

Proof. We shall exhibit a sequence $\left(s_{n}\right)$ in $\mathbf{M}_{2}(\mathbf{C})$ which is positive definite but not of positive type. The corresponding linear mapping then has the properties claimed.

Define $a_{n}=2^{(n+2) !}$ for $n \geq 0$ and

$$
\begin{gathered}
s_{0}=\left(\begin{array}{ll}
4 & 0 \\
0 & 1
\end{array}\right), \quad s_{1}=\left(\begin{array}{ll}
0 & 2 \\
2 & 0
\end{array}\right), \quad s_{2}=\left(\begin{array}{ll}
1 & 0 \\
0 & 4
\end{array}\right), \\
s_{2 n-1}=\left(\begin{array}{ll}
0 & 0 \\
0 & 0
\end{array}\right), \quad s_{2 n}=\left(\begin{array}{cc}
a_{n} & 0 \\
0 & a_{n}
\end{array}\right)
\end{gathered}
$$

for $n \geq 2$. To show that $\left(s_{n}\right)$ is positive definite it suffices to show that the determinant

$$
D_{n}(\xi)=\left|\begin{array}{ccc}
\left\langle s_{0} \xi, \xi\right\rangle & \ldots & \left\langle s_{n} \xi, \xi\right\rangle \\
\vdots & & \vdots \\
\left\langle s_{n} \xi, \xi\right\rangle & \ldots & \left\langle s_{2 n} \xi, \xi\right\rangle
\end{array}\right|
$$

is positive for each $n \geq 0$ and each $\xi$ in the unit sphere of $\mathbf{C}^{2}$. So suppose $\xi=\left(\xi_{1}, \xi_{2}\right)$ with $\left|\xi_{1}\right|^{2}+\left|\xi_{2}\right|^{2}=1$. Then

$$
\begin{gathered}
D_{0}(\xi)=4\left|\xi_{1}\right|^{2}+\left|\xi_{2}\right|^{2} \geq 1, \\
D_{1}(\xi)=\left(4\left|\xi_{1}\right|^{2}+\left|\xi_{2}\right|^{2}\right)\left(\left|\xi_{1}\right|^{2}+4\left|\xi_{2}\right|^{2}\right)-\left(4 \operatorname{Re}\left(\overline{\xi_{1}} \xi_{2}\right)\right)^{2} \\
\geq 4\left|\xi_{1}\right|^{4}+4\left|\xi_{2}\right|^{4} \geq 2\left(\left|\xi_{1}\right|^{2}+\left|\xi_{2}\right|^{2}\right)^{2} \geq 1 .
\end{gathered}
$$

Now assume $n \geq 2$ and $D_{n-1}(\xi) \geq 1$. Expand $D_{n}(\xi)$ completely, then collect the $n$ ! terms containing $\left\langle s_{2 n} \xi, \xi\right\rangle$ into one. For the remaining $(n+1) !-n$ ! terms use

$$
\left|\left\langle s_{k} \xi, \xi\right\rangle\right| \leq\left\|s_{k}\right\| \leq \max \left\{4, a_{2}, \ldots, a_{n-1}\right\} \leq a_{n-1}
$$

$(k=0, \ldots, 2 n-1)$ to get a bound. The result is

$$
\begin{aligned}
D_{n}(\xi) & \geq\left\langle s_{2 n} \xi, \xi\right\rangle D_{n-1}(\xi)-((n+1) !-n !) a_{n-1}^{n+1} \\
& \geq a_{n}-(n+1) ! a_{n-1}^{n+1}=2^{(n+1)(n+1) !}\left(2^{(n+1) !}-(n+1) !\right) \geq 1 .
\end{aligned}
$$


So $\left(s_{n}\right)$ is positive definite. To see that $\left(s_{n}\right)$ is not of positive type, take $\xi_{0}=$ $(0,1)$ and $\xi_{1}=(-1,0)$ and verify $\sum_{j, k=0}^{1}\left\langle s_{j+k} \xi_{k}, \xi_{j}\right\rangle=-2$.

A sequence $\left(s_{n}\right)$ in $\mathbf{B}(\mathscr{H})$ is a(n operator) moment sequence if there is a measure $\mu$, defined on the Borel field $\mathscr{B}(\mathbf{R})$ and taking values in $\mathbf{B}(\mathscr{H})_{+}$, such that

$$
\left\langle s_{n} \xi, \eta\right\rangle=\int_{\mathbf{R}} x^{n} d\langle\mu \xi, \eta\rangle(x)
$$

for all $\xi, \eta \in \mathscr{H}$ and $n \geq 0$. Here $\langle\mu \xi, \eta\rangle$ is the complex measure defined by $\langle\mu \xi, \eta\rangle(E)=\langle\mu(E) \xi, \eta\rangle, E \in \mathscr{B}(\mathbf{R})$. (By definition, $\mu: \mathscr{B}(\mathbf{R}) \rightarrow \mathbf{B}(\mathscr{H})_{+}$is a measure if and only if $\langle\mu \xi, \xi\rangle$ is a measure for each $\xi \in \mathscr{H}$.) By Hamburger's Theorem ([4, 6.2.2] or [1]), a sequence $\left(s_{n}\right)_{n \geq 0}$ in $\mathbf{R}$ is a moment sequence if and only if it is positive definite. General references for this classical moment problem are [1, 10]; see [4] for the generalization to semigroups other than the integers. For an arbitrary Hilbert space $\mathscr{H}$ moment sequences in $\mathbf{B}(\mathscr{H})$ are precisely the sequences of positive type; see, for example, [9].

Suppose $\left(s_{n}\right)$ is a positive definite sequence in $\mathbf{B}(\mathscr{H})$. For each $\xi \in \mathscr{H}$ the sequence $\left(\left\langle s_{n} \xi, \xi\right\rangle\right)$ is positive definite, so one may choose a measure $\lambda_{\xi}$ on $\mathbf{R}$ such that

$$
\left\langle s_{n} \xi, \xi\right\rangle=\int_{\mathbf{R}} x^{n} d \lambda_{\xi}(x)
$$

for all $n \geq 0$. If a $\mathbf{B}(\mathscr{H})_{+}$-valued measure $\mu$ exists which satisfies

$$
\langle\mu \xi, \xi\rangle=\lambda_{\xi}, \quad \xi \in \mathscr{H},
$$

then $\mu$ satisfies equation (2) (by polarization), so $\left(s_{n}\right)$ is a moment sequence. The existence of such a measure $\mu$, however, cannot be taken for granted. Indeed, if (4) holds then

$$
\begin{aligned}
\lambda_{\xi+\eta}+\lambda_{\xi-\eta} & =\langle\mu(\xi+\eta), \xi+\eta\rangle+\langle\mu(\xi-\eta), \xi-\eta\rangle \\
& =2(\langle\mu \xi, \xi\rangle+\langle\mu \eta, \eta\rangle)=2\left(\lambda_{\xi}+\lambda_{\eta}\right)
\end{aligned}
$$

and

$$
\lambda_{c \xi}=\langle\mu(c \xi), c \xi\rangle=|c|^{2}\langle\mu \xi, \xi\rangle=|c|^{2} \lambda_{\xi}
$$

for all $\xi, \eta \in \mathscr{H}$ and $c \in \mathbf{C}$. In short, the family $\left(\lambda_{\xi}\right)_{\xi \in \mathscr{L}}$ has to satisfy the parallelogram law

$$
\lambda_{\xi+\eta}+\lambda_{\xi-\eta}=2\left(\lambda_{\xi}+\lambda_{\eta}\right)
$$

$(\xi, \eta \in \mathscr{H})$ and the rule of homogeneity

$$
\lambda_{c \xi}=|c|^{2} \lambda_{\xi}
$$

$(\xi \in \mathscr{H}$ and $c \in \mathbf{C})$. These conditions are also sufficient. To see this, suppose they hold. For each Borel set $E$ in $\mathbf{R}$ the family $\left(\lambda_{\xi}(E)\right)_{\xi \in \mathscr{Z}}$ satisfies the parallelogram law and the rule of homogeneity (same as above, only for scalars instead of measures), and it is a matter of algebra that there is a unique sesquilinear form $f_{E}$ on $\mathscr{H}$ such that $\lambda_{\xi}(E)=f_{E}(\xi, \xi)$ for all $\xi \in \mathscr{H}$. Now 
$f_{E}$ is positive since the $\lambda_{\xi}$ are positive measures and is bounded since (by (3))

$$
f_{E}(\xi, \xi)=\lambda_{\xi}(E) \leq \lambda_{\xi}(\mathbf{R})=\left\langle s_{0} \xi, \xi\right\rangle \leq\|s\|\|\xi\|^{2} .
$$

Hence there is a unique $\mu(E) \in \mathbf{B}(\mathscr{H})_{+}$such that $f_{E}(\xi, \eta)=\langle\mu(E) \xi, \eta\rangle$ for all $\xi, \eta \in \mathscr{H}$. In particular $\lambda_{\xi}(E)=\langle\mu(E) \xi, \xi\rangle$ for all $\xi \in \mathscr{H}$. This being so for all $E \in \mathscr{B}(\mathbf{R})$, it follows that $\mu$ is a measure and that (4) holds.

A moment sequence $\left(s_{n}\right)$ in $\mathbf{B}(\mathscr{H})$ is determinate if only one measure $\mu$ satisfies (2).

Theorem 2. Let $\left(s_{n}\right)$ be a positive definite sequence in $\mathbf{B}(\mathscr{H})$, and assume that for all $\xi, \eta \in \mathscr{H}$ the scalar moment sequence $\left(\left\langle s_{n} \xi, \xi\right\rangle+\left\langle s_{n} \eta, \eta\right\rangle\right)_{n \geq 0}$ is determinate. Then $\left(s_{n}\right)$ is a determinate moment sequence.

Proof. For each $\xi \in \mathscr{H}$ the moment sequence $\left(\left\langle s_{n} \xi, \xi\right\rangle\right)$ is determinate (take $\eta=0$ in the statement of the theorem); let $\lambda_{\xi}$ be the unique measure for which (3) holds. By the uniqueness of $\lambda_{\xi}$, any measure $\mu$ satisfying (2) must satisfy (4). By polarization it follows that $\left(s_{n}\right)$, if a moment sequence, is determinate.

For $\xi, \eta \in \mathscr{H}$ we have

$$
\begin{gathered}
\int x^{n} d \lambda_{\xi+\eta}(x)+\int x^{n} d \lambda_{\xi-\eta}(x)=\left\langle s_{n}(\xi+\eta), \xi+\eta\right\rangle+\left\langle s_{n}(\xi-\eta), \xi-\eta\right\rangle \\
=2\left\langle s_{n} \xi, \xi\right\rangle+2\left\langle s_{n} \eta, \eta\right\rangle=2 \int x^{n} d \lambda_{\xi}(x)+2 \int x^{n} d \lambda_{\eta}(x)
\end{gathered}
$$

$(n \geq 0)$. By the determinacy hypothesis it follows that (5) holds; (6) is gotten by a similar argument. This proves the existence of a $\mathbf{B}(\mathscr{H})_{+}$-valued measure $\mu$ satisfying (4) and hence (by (3) and polarization) (2), thus completing the proof of the theorem.

Recall the following two criteria of Carleman for determinacy in the classical moment problem: First, suppose $\left(s_{n}\right)$ is a sequence of reals such that the determinants

$$
D_{n}=\left|\begin{array}{ccc}
s_{0} & \ldots & s_{n} \\
\vdots & & \vdots \\
s_{n} & \ldots & s_{2 n}
\end{array}\right|
$$

$(n \geq 0)$ are positive. (In particular, $\left(s_{n}\right)$ is positive definite, hence a moment sequence). If

$$
\sum_{n=2}^{\infty} \frac{D_{n-1}}{\sqrt{D_{n-2} D_{n}}}=\infty
$$

then $\left(s_{n}\right)$ is determinate.

Second, every scalar moment sequence $\left(s_{n}\right)$ satisfying

$$
\sum_{n=1}^{\infty} \frac{1}{\sqrt[2 n]{s_{2 n}}}=\infty
$$

is determinate (see [1]). 
Corollary 1. Let $\left(s_{n}\right)$ be a positive definite sequence in $\mathbf{B}(\mathscr{H})$, and suppose

$$
\sum_{n=1}^{\infty} \frac{1}{\sqrt[2 n]{\left\|s_{2 n}\right\|}}=\infty
$$

Then $\left(s_{n}\right)$ is a determinate moment sequence.

Proof. Given $\xi, \eta \in \mathscr{H}$, apply the second Carleman criterion to the sequence $\left(\left\langle s_{n} \xi, \xi\right\rangle+\left\langle s_{n} \eta, \eta\right\rangle\right)$.

Though conceivably $\left(\left\langle s_{n} \xi, \xi\right\rangle+\left\langle s_{n} \eta, \eta\right\rangle\right)$ might be replaced by just $\left(\left\langle s_{n} \xi, \xi\right\rangle\right)$ in Theorem 2, a proof of this can hardly be simple. Certainly the naïve approach is doomed to failure; the sum of two determinate moment sequences need not be determinate. (In fact, every (scalar) moment sequence is the sum of two determinate ones. To see this, consider any moment sequence $\left(s_{n}\right)$. If $\left(s_{n}\right)$ is determinate, it is the sum of the two determinate moment sequences $(0)$ and $\left(s_{n}\right)$. Otherwise, choose a Nevanlinna extremal measure $\mu$ of which $\left(s_{n}\right)$ is the moment sequence (see [1]). Such a measure has the form $\mu=\sum_{n=1}^{\infty} a_{n} \varepsilon_{x_{n}}$ where the $x_{n}$ are distinct reals, $a_{n}>0$, and $\varepsilon_{x_{n}}$ denotes the Dirac measure at $x_{n}$. Moreover, $\mu-a_{n} \varepsilon_{x_{n}}$ is determinate for each $n$ [3]. So $\left(s_{n}\right)$ is the sum of the moment sequences of $a_{1} \varepsilon_{x_{1}}$ and $\mu-a_{1} \varepsilon_{x_{1}}$; both of which are determinate.) Determinacy of a great many of the scalar moment sequences $\left(\left\langle s_{n} \xi, \xi\right\rangle\right)$ is not sufficient.

Theorem 3. There is a positive definite sequence $\left(s_{n}\right)_{n \geq 0}$ in $\mathbf{B}\left(\mathbf{C}^{2}\right)$ such that the scalar moment sequence $\left(\left\langle s_{n} \xi, \xi\right\rangle\right)$ is determinate for each $\xi$ in a dense $G_{\delta}$ in $\mathrm{C}^{2}$; yet $\left(s_{n}\right)$ is not a moment sequence.

Proof. Let $\Sigma$ be the unit sphere in $\mathbf{C}^{2}, \Phi$ a countable dense set in $\Sigma$. Choose a sequence $\left(\varphi_{n}\right)_{n \geq 2}$ in $\Phi$ in which each element of $\Phi$ appears infinitely often.

We shall construct the sequence $\left(s_{n}\right)$ by induction, keeping the determinants (1) positive for all $n$ and all $\xi \in \Sigma$ (hence for all $\xi \in \mathbf{C}^{2} \backslash\{0\}$ ), thus making sure that $\left(s_{n}\right)$ will be positive definite. As in the proof of Theorem 1, beginning the construction with

$$
s_{0}=\left(\begin{array}{ll}
4 & 0 \\
0 & 1
\end{array}\right), \quad s_{1}=\left(\begin{array}{ll}
0 & 2 \\
2 & 0
\end{array}\right), \quad s_{2}=\left(\begin{array}{ll}
1 & 0 \\
0 & 4
\end{array}\right)
$$

ensures that the full sequence will not be of positive type (i.e., not a moment sequence).

Suppose that $n \geq 2$ and that $s_{0}, \ldots, s_{2 n-2}$ have been chosen properly. Put $s_{2 n-1}=0$ and define a function $F_{n}$ on $C^{2}$ by the condition that

$$
\left|\begin{array}{cccc}
\left\langle s_{0} \xi, \xi\right\rangle & \ldots & \left\langle s_{n-1} \xi, \xi\right\rangle & \left\langle s_{n} \xi, \xi\right\rangle \\
\vdots & & \vdots & \vdots \\
\left\langle s_{n-1} \xi, \xi\right\rangle & \ldots & \left\langle s_{2 n-2} \xi, \xi\right\rangle & \left\langle s_{2 n-1} \xi, \xi\right\rangle \\
\left\langle s_{n} \xi, \xi\right\rangle & \ldots & \left\langle s_{2 n-1} \xi, \xi\right\rangle & t
\end{array}\right|=D_{n-1}(\xi) t+F_{n}(\xi)
$$

for all $\xi \in \mathbf{C}^{2}$ and $t \in \mathbf{R}$. Let $p: \mathbf{C}^{2} \rightarrow \mathbf{C} \varphi_{n}$ be the orthogonal projection. Since $D_{n-1}\left(\varphi_{n}\right)>0$ by assumption, we may choose $a \in \mathbf{R}$ such that

$$
0<D_{n-1}\left(\varphi_{n}\right) a+F_{n}\left(\varphi_{n}\right)<\frac{D_{n-1}\left(\varphi_{n}\right)^{2}}{D_{n-2}\left(\varphi_{n}\right)} \text {. }
$$


Note that $a>0$ since the matrix in (7) becomes strictly positive definite upon substituting $a$ for $t$ and $\varphi_{n}$ for $\xi$. The obvious homogeneity properties of $D_{n-1}$ and $F_{n}$ imply that

$$
D_{n-1}(\xi) a+F_{n}(\xi)=D_{n-1}\left(\varphi_{n}\right) a+F_{n}\left(\varphi_{n}\right)
$$

for each $\xi \in \mathbf{T} \varphi_{n}$ where $\mathbf{T}=\{c \in \mathbf{C}|| c \mid=1\}$. Since $D_{n-1}$ and $F_{n}$ are continuous, it follows that

$$
D_{n-1}(\xi)\|p \xi\|^{2} a+F_{n}(\xi)>0
$$

for all $\xi$ in some neighbourhood of $T \varphi_{n}$. On the remainder of $\Sigma$, the function $\xi \rightarrow\|(1-p) \xi\|$ is bounded below away from zero. Hence, if $b>0$ is chosen large enough, then

$$
D_{n-1}(\xi)\left(a\|p \xi\|^{2}+b\|(1-p) \xi\|^{2}\right)+F_{n}(\xi)>0
$$

for all $\xi \in \Sigma$. Defining $s_{2 n}=a p+b(1-p)$ we have

$$
\begin{aligned}
D_{n}(\xi) & =D_{n-1}(\xi)\left\langle s_{2 n} \xi, \xi\right\rangle+F_{n}(\xi) \\
& =D_{n-1}(\xi)\left(a\|p \xi\|^{2}+b\|(1-p) \xi\|^{2}\right)+F_{n}(\xi)>0
\end{aligned}
$$

for all $\xi \in \Sigma$. Note that

$$
\begin{aligned}
D_{n}\left(\varphi_{n}\right) & =D_{n-1}\left(\varphi_{n}\right)\left\langle s_{2 n} \varphi_{n}, \varphi_{n}\right\rangle+F_{n}\left(\varphi_{n}\right) \\
& =D_{n-1}\left(\varphi_{n}\right) a+F_{n}\left(\varphi_{n}\right)<\frac{D_{n-1}\left(\varphi_{n}\right)^{2}}{D_{n-2}\left(\varphi_{n}\right)} .
\end{aligned}
$$

For each $n \geq 2$ the set

$$
G_{n}=\left\{\xi \in \mathbf{C}^{2} \backslash\{0\} \mid D_{n-2}(\xi) D_{n}(\xi)<D_{n-1}(\xi)^{2}\right\}
$$

is open, contains $\varphi_{n}$ according to (8), and is stable under multiplication by nonzero scalars (by the homogeneity properties of the functions $D_{k}$ ). The $G_{\delta^{-}}$ set

$$
A=\bigcap_{n=2}^{\infty} \bigcup_{m=n}^{\infty} G_{m}
$$

contains $\Phi$ and is stable under multiplication by nonzero scalars; so $A$ is dense in $\mathbf{C}^{2}$. If $\xi \in A$, then $D_{n-2}(\xi) D_{n}(\xi)<D_{n-1}(\xi)^{2}$ for infinitely many $n$, hence

$$
\sum_{n=2}^{\infty} \frac{D_{n-1}(\xi)}{\sqrt{D_{n-2}(\xi) D_{n}(\xi)}}=\infty
$$

By the Carleman criterion it follows that the moment sequence $\left(\left\langle s_{n} \xi, \xi\right\rangle\right)_{n \geq 0}$ is determinate.

\section{ACKNOWLEDGMENTS}

The author is indebted to Professor Christian Berg, who suggested the problem and pointed out a simplification of what was to become Theorem 2; to Professor Søren Asmussen, who introduced him to $\mathrm{LT}_{\mathrm{E}} \mathrm{X}$; and to $\mathrm{M}$. and D., who provided shelter and a Macintosh ${ }^{T M}$ during one sunny Easter. 


\section{REFERENCES}

1. N. I. Akhiezer, The classical moment problem, Oliver \& Boyd, Edinburgh, 1965.

2. W. B. Arveson, Subalgebras of $C^{*}$-algebras, Acta Math. 123 (1969), 141-224.

3. C. Berg and J. P. R. Christensen, Density questions in the classical theory of moments, Ann. Inst. Fourier (Grenoble) 31 (1981), 99-114.

4. C. Berg, J. P. R. Christensen, and P. Ressel, Harmonic analysis on semigroups, SpringerVerlag, New York, Berlin, Heidelberg, and Tokyo, 1984.

5. D. E. Evans, Quantum dynamical semigroups, symmetry groups and locality, Acta Appl. Math. 2 (1984), 333-352.

6. D. E. Evans and J. T. Lewis, Dilations of irreversible evolutions in algebraic quantum theory. Comm. Dublin Inst. Adv. Stud. Ser. A, vol. 24, Inst. Adv. Stud., Dublin, 1977.

7. J. S. MacNerney, Hermitean moment sequences, Trans. Amer. Math. Soc. 103 (1962), 4581.

8. W. Mlak, Dilations of Hilbert space operators (general theory), Dissertationes Math. (Rozprawy Mat.) 153 (1978), 1-61.

9. K. Schmüdgen, On a generalization of the classical moment problem, J. Math. Anal. Appl. 125 (1987), 461-470.

10. J. A. Shohat and J. D. Tamarkin, The problem of moments, Amer. Math. Soc., Providence, RI, 1943.

11. W. F. Stinespring, Positive functions on $C^{*}$-algebras, Proc. Amer. Math. Soc. 6 (1955), 211-216.

12. B. Sz.-Nagy, A moment problem for self-adjoint operators, Acta. Sci. Math. (Szeged) 3 (1952), 285-292.

13. $ـ$ Extension of linear transformations in Hilbert space which extend beyond this space, Ungar, New York, 1960.

Department of Mathematics and Computer Science, Institute of Electronic Systems, Aalborg University, Fredrik Bajersvej 7, DK-9220 Aalborg $\varnothing$, Denmark

Current address: Nandrupsvej 7, DK-2000 Frederiksberg C, Denmark 